\title{
The Influence of Fiber Laser Pulse Processing on Coercivity and Nanohardness of Fe-3.2Si Grain-Oriented Electrical Steel in relation with its Surface Changes and Magnetic Domains Modifications
}

\author{
V. PuchÝ*, L. Falat, F. Kováč, I. Petryshynets, R. Džunda And M. ŠEbek \\ Institute of Materials Research, Slovak Academy of Sciences, Watsonova 47, 04001 Košice, Slovak Republic \\ (Received December 6, 2015; in final form April 14, 2017) \\ Fiber laser scribing of Fe-3.2Si grain-oriented electrical steel was carried out using three different laser pulse \\ regimes, namely a single pulse laser regime, multipulse laser regime and multipulse laser regime with modulation \\ of laser pulses. The samples were tested for coercivity and nanohardness before and after laser processing. Clear \\ improvements in soft magnetic characteristics were observed for all the regimes using the lowest single pulse energy \\ of $0.6 \mathrm{~mJ}$. The best improvement i.e. the most significant decrease in coercivity was obtained after the regime \\ with 5 pulses. Further increasing in both pulse energy and pulse number resulted only in detrimental effects on \\ both magnetic and mechanical characteristics. Introduction of $50 \mathrm{kHz}$ pulse modulation within 100-pulse laser \\ regimes resulted in lower coercivity values in comparison with the same regimes without modulation. The major \\ effects responsible for the observed improvement are related to optimal refinement and rearrangement of magnetic \\ domains determined by the balance of laser-induced residual stresses and plastic deformation at the surface of the \\ treated material. Thus the fiber laser pulse processing has been recognised as a useful and energy efficient tool in \\ obtaining electrical steels for power transformers with lower core losses.
}

DOI: 10.12693/APhysPolA.131.1445

PACS/topics: $61.80 . \mathrm{Ba}, 81.05 . \mathrm{Bx}, 81.40 . \mathrm{Rs}, 81.40 .-\mathrm{z}, 75.60 . \mathrm{Ch}, 79.20 . \mathrm{Eb}$

\section{Introduction}

The need for energy saving is a constant impulse for new developments and it also evokes considerations about the performance losses in devices such as electric motors, transformers and transmitters. These losses have to be reduced in order to save energy, reduce the heat and limit the frequency dependency of the magnetic properties. The promising results have been shown in grainoriented (GO) electrical steel sheets through the refinement of magnetic domain structure as a result of mechanical and/or thermal stresses implementation $[1,2]$. The desired effects can be achieved by mechanical scribing/scratching, electrical discharge machining, plasma flame treatment, chemical etching and also by laser scribing [3-9]. The laser scribing technique is based on rapid laser-induced increase in temperature on the sheet surface causing residual stress during subsequent cooling that refines the domains. The mechanisms responsible for the improvement of soft magnetic properties include magnetic domain refinement, stress relaxation, and inhibition of domain-wall movement [10]. The commercially available laser scribing systems have been optimized typically for other applications and other materials such as scribing of crystalline $\mathrm{Si}$, resistor trimming, or circuit repair [11, 12]. In contrast to laser welding [13] or surface alloying by laser melt deposition [14], the laser

\footnotetext{
*corresponding author; e-mail: vpuchy@saske.sk
}

scribing may apply only a very low input power (e.g. up to $100 \mathrm{~W}$ ), thus avoiding surface melting related deterioration of the treated material.

Our previous work [15] was focused on optimizing the laser scribing conditions of $\mathrm{Fe}-3.2 \mathrm{Si}$ electrical steel sheets for single pulse laser regimes. The present work represents an extended and continuing research study to our preliminary investigation [16] of the effects of multipulse fiber laser processing on magnetic domains structure and surface changes of the same material in correlation with its resulting soft magnetic and nanohardness characteristics.

\section{Experimental material and procedure}

The Fe-3.2Si electrical GO steel of M165-35S grade was produced by ArcelorMittal Frýdek-Místek a.s. and the samples with dimensions of $30 \mathrm{~mm} \times 10 \mathrm{~mm}$ $\times 0.35 \mathrm{~mm}$ were investigated in present study. The chemical composition of the experimental material is listed in Table I.

TABLE I

Chemical composition [wt\%] of investigated M165-35S grade electrical GO steel.

\begin{tabular}{c|c|c|c|c|c|c|c|c}
\hline \hline $\mathrm{Si}$ & $\mathrm{C}$ & $\mathrm{Mn}$ & $\mathrm{S}$ & $\mathrm{Cu}$ & $\mathrm{P}$ & $\mathrm{Al}$ & $\mathrm{N}$ & $\mathrm{Fe}$ \\
\hline 3.15 & 0.006 & 0.21 & 0.009 & 0.44 & 0.007 & 0.013 & 0.011 & bal.
\end{tabular}

All samples were prepared by laser cutting with oxygen assist gas for better cutting performance avoiding introduction of residual stresses. Afterwards, the samples 
were annealed in nitrogen atmosphere and then electrolytically polished in a solution of $\mathrm{CH}_{3} \mathrm{COOH}$ and $\mathrm{HClO}_{4}$ (20:1 per volume). The electro-polishing conditions were set to $50 \mathrm{~V}$ and $20^{\circ} \mathrm{C}$ using Lectropol-5 electrolytic polishing machine and the final cleaning of the prepared mirror-finish steel sheet surfaces was carried out in deionized water and ethanol. In this way, sufficiently smooth surfaces of studied GO silicon steel sheets were prepared for magnetic domain observation. This procedure was also used to avoid the generation of residual stresses which might otherwise appear in the case of mechanical polishing. The samples after preparation were mounted in a sample holder and set into the experimental laser workstation TRUMPF 3003 with laser source TruFiber400. The samples were laser treated in air using programmable focused optic head. The fiber laser radiation with a wavelength of $1064 \mathrm{~nm}$ and focus on the sample surface was used. The laser scribing conditions included variations in pulse energy and total number of applied pulses. The pulse frequency, pulse separation distance (laser spot spacing) and scan separation distance (scribe lines spacing) were $200 \mathrm{~Hz}, 0.2 \mathrm{~mm}$, and $5 \mathrm{~mm}$, respectively. The scan direction was oriented perpendicularly to the rolling direction of investigated material and three different laser pulse regimes were used, namely a single pulse laser regime, multipulse laser regime and multipulse laser regime with modulation of laser pulses (Fig. 1).

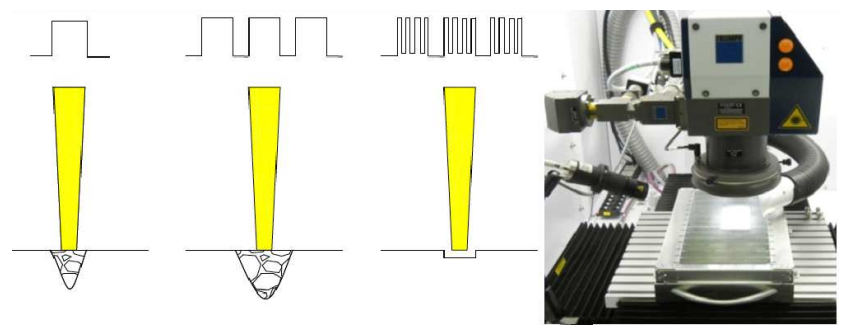

Fig. 1. Scheme of individual regimes of applied laser processing and a photograph of experimental laser workstation.

The laser spot diameter on the surface of the sample was approximately $30 \mu \mathrm{m}$. The input power of laser beam varied from $12 \mathrm{~W}$ to $100 \mathrm{~W}$ with a pulse duration from $50 \mu \mathrm{s}$ to $500 \mu \mathrm{s}$ resulting in pulse energies from $0.6 \mathrm{~mJ}$ up to $50 \mathrm{~mJ}$. The total number of applied pulses included 1 , $5,10,50$, and 100 pulses. In addition, the 100-pulse regime was examined also for a pulse frequency modulation of $50 \mathrm{kHz}$. The microstructure and morphology of laser treated surfaces were observed by scanning electron microscope (SEM) JEOL JSM-7000F. The measurement of coercivity was performed using "Oersted type" coercivitymeter KPS-1C. The magnetic domains were observed using four visualizing techniques, namely the Bitter microscopy, magnetic force microscopy (MFM), the Kerr microscopy, and also SEM microscopy. The samples for the Bitter observation technique were prepared by electrolytic polishing and coating with magnetic colloid suspension Ferrofluid [17]. Afterwards, the magnetic domains were observed in the Nomarski differential interference contrast (DIC) using optical microscope Olympus GX71. For the MFM observation an AFM microscope Dimension Icon (Bruker) with Bruker $\mathrm{Co} / \mathrm{Cr}$ tip was used, similarly like in study [18], focused on imaging of magnetic domains in spherical Fe-Si powder. The Kerr scanning magneto-optical observation was performed using the Kerr microscope EVICO MAGNETICS. The observation of magnetic domains by SEM was carried out at specific conditions (tilt angle $0^{\circ}$, voltage $10 \mathrm{kV}$, probe current $7 \mathrm{nA}$ ) that were also used in [19]. The domain width was measured with the aid of the software Image $\mathrm{J}$ for Windows. A minimum of 50 readings was taken to measure the domain width of each material state and the results were treated statistically. Nanohardness measurements were performed in centre of the laser spots using nanohardness tester Agilent G200 with applied load of $50 \mathrm{mN}$.

\section{Results and discussion}

Figure 2 shows typical cross-sectional metallographic images of laser affected zones created by three different single pulse laser regimes with pulse energy of $0.6,5$, and $50 \mathrm{~mJ}$, respectively.

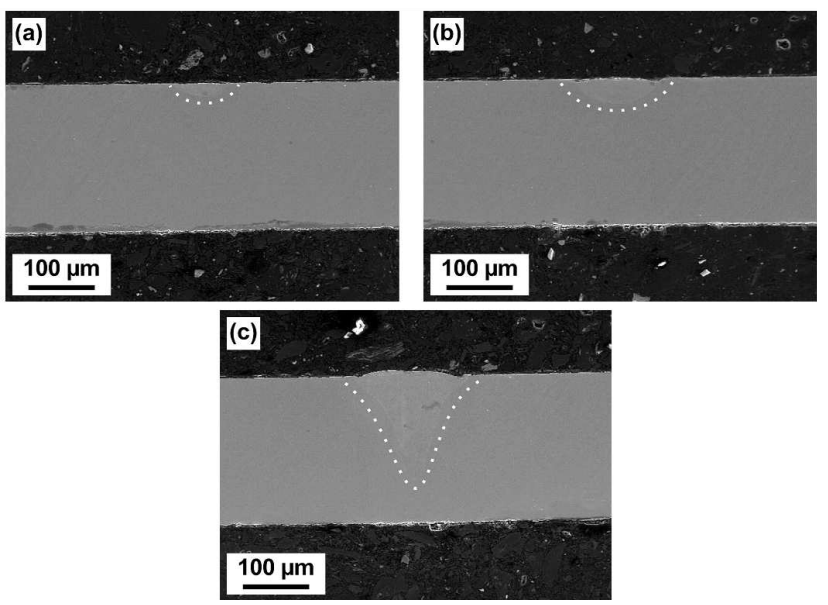

Fig. 2. SEM secondary electrons visualization of metallographic cross-sections showing laser affected zones formed after single pulse laser regimes with pulse energy of: (a) $0.6 \mathrm{~mJ}$, (b) $5 \mathrm{~mJ}$, (c) $50 \mathrm{~mJ}$.

The single pulses with input energy of $0.6 \mathrm{~mJ}$ and $5 \mathrm{~mJ}$ (Fig. 2a,b) caused only a negligible melting effect on the surface of the material. On the other hand, the highest applied single pulse energy caused a significant melting effect with high depth of penetration (Fig. 2c).

Figure 3 demonstrates the influence of number and modulation of laser pulses in regimes with $50 \mathrm{~mJ}$ single pulse energy on the material surface. For a single pulse regime (Fig. 3a) the surface ablation is negligible, whereas the multipulse laser regimes with 100 pulses (Fig. 3b) and 100 modulated pulses (Fig. 3c) already resulted in significant surface ablation.

Thus it can be summarized that with increase of number of pulses and introduction of pulse modulation, 


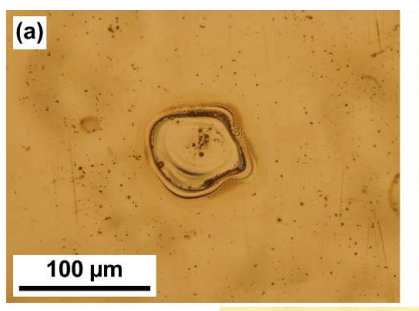

(b)

(c)

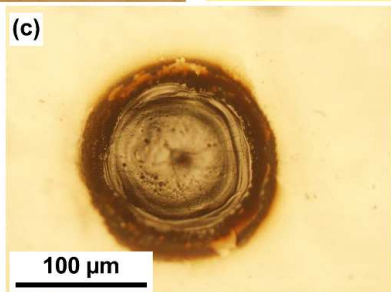

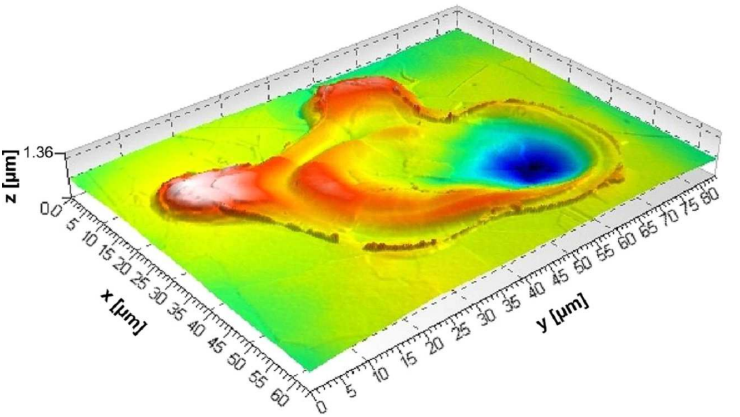

Fig. 5. Laser scanning confocal visualization of laser spot created by multipulse laser regime with 100 modulated pulses with single pulse energy of $0.6 \mathrm{~mJ}$.

TABLE II

Soft magnetic and mechanical characteristics of investigated $\mathrm{Fe}-3.2 \mathrm{Si}$ GO electrical steel in dependence of applied fiber laser pulse processing conditions.

\begin{tabular}{|c|c|c|c|c|c|c|}
\hline 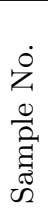 & 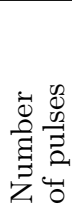 & 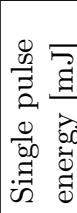 & 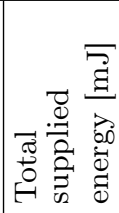 & 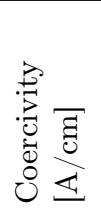 & 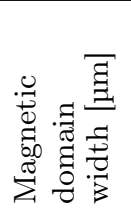 & 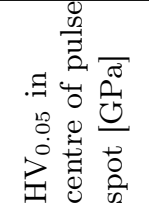 \\
\hline 1 & 0 & 0 & 0 & 0.057 & $45 \pm 4.2$ & $4.2 \pm 0.1$ \\
\hline 2 & 1 & 0.6 & 0.6 & 0.039 & $16 \pm 2.1$ & $3.96 \pm 0.24$ \\
\hline 3 & 1 & 5 & 5 & 0.062 & $39 \pm 3.8$ & $3.91 \pm 0.17$ \\
\hline 4 & 1 & 50 & 50 & 0.080 & $50 \pm 5.5$ & $3.85 \pm 0.14$ \\
\hline 5 & 5 & 0.6 & 3 & 0.035 & $15 \pm 2.0$ & $3.97 \pm 0.26$ \\
\hline 6 & 5 & 5 & 25 & 0.065 & $41 \pm 3.9$ & $3.90 \pm 0.18$ \\
\hline 7 & 5 & 50 & 250 & 0.070 & $43 \pm 4.3$ & $3.83 \pm 0.15$ \\
\hline 8 & 10 & 0.6 & 6 & 0.037 & $15 \pm 1.9$ & $3.98 \pm 0.27$ \\
\hline 9 & 10 & 5 & 50 & 0.064 & $40 \pm 3.7$ & $3.92 \pm 0.18$ \\
\hline 10 & 10 & 50 & 500 & 0.069 & $42 \pm 4.2$ & $3.84 \pm 0.16$ \\
\hline 11 & 50 & 0.6 & 30 & 0.038 & $16 \pm 2.5$ & $3.99 \pm 0.29$ \\
\hline 12 & 50 & 5 & 250 & 0.070 & $43 \pm 4.3$ & $3.94 \pm 0.19$ \\
\hline 13 & 50 & 50 & 2500 & 0.069 & $43 \pm 4.7$ & $3.86 \pm 0.17$ \\
\hline 14 & 100 & 0.6 & 60 & 0.055 & $23 \pm 2.9$ & $3.99 \pm 0.33$ \\
\hline 15 & 100 & 5 & 500 & 0.084 & $38 \pm 3.6$ & $3.94 \pm 0.27$ \\
\hline 16 & 100 & 50 & 5000 & $>0.30$ & $120 \pm 9.1$ & $3.83 \pm 0.16$ \\
\hline 17 & $100^{*}$ & 0.6 & 60 & 0.050 & $29 \pm 3.1$ & n.d. \\
\hline 18 & $100^{*}$ & 5 & 500 & 0.080 & $52 \pm 6.5$ & n.d. \\
\hline 19 & $100^{*}$ & 50 & 5000 & 0.120 & $75 \pm 8.1$ & n.d. \\
\hline
\end{tabular}

* pulse frequency modulation $50 \mathrm{kHz}$,

n.d. - not determined due to significant ablation. $0.6 \mathrm{~mJ}$ applied in multipulse laser regime with $100 \mathrm{mo}$ dulated pulses (Fig. 4a) resulted in small ablation effect without the presence of cracks within the laser spot. However, the higher single pulse energy values (Fig. 4b,c) in the same types of laser processing regimes resulted in significant ablation effects and the occurrence of surface cracks in the areas of laser spots. The detailed visualization of the ablation crater obtained after the 100 modulated pulses regime with $0.6 \mathrm{~mJ}$ single pulse energy is documented in Fig. 5.

From this image it is evident that the surface irradiated by frequency modulated pulses exhibits clear height differences due to the mass transfer occurred. This effect was caused due to modulated pulse energy divided into many short pulses which generated short microsecond pulsed ablation. This mechanism has also been investigated by several other authors [20,21]. They were using shorter pulses in the nanosecond range. However, in our case, we get similar results with the microsecond modulated multipulse regime. The summary of the measured properties is presented in Table II. The comparison of average domain width values before and after the laser pulse treatment shows the obtained changes due to laser processing 
conditions variation. It should be noted that the observed differences in values of magnetic domain width included either their refinement or widening for individual laser pulse regimes (Table II). As also found by other authors $[22,23]$ the core losses depend on the domain size as well as on the domain wall mobility. This implies that in certain situation the core losses may be even higher after the laser modification which has also been confirmed by partial results of present study. As shown in Table II, the revealed coercivity increase despite decreasing domain width can likely be related to the decreased mobility of primary magnetic domains in the vicinity of laser spots due to the presence of detrimental labyrinth domains. The results presented in Table II also clearly show that the application of all fiber laser pulse regimes resulted in deterioration of local mechanical properties characterised by decreasing nanohardness values in centre of laser spots.

Figures 6-9 show variations in magnetic domain structures induced by laser treatment using several visualization techniques. Figure 6 demonstrates the effect of $5 \mathrm{~mJ}$ single pulse laser regime on the refinement of magnetic domains visualized by the Bitter microscopy. Figure $6 \mathrm{a}$ shows exclusively the primary $180^{\circ}$ (slab) domains in laser untreated sample, whereas Fig. $6 \mathrm{~b}$ indicates the formation of labyrinth subdomains around the laser spots.
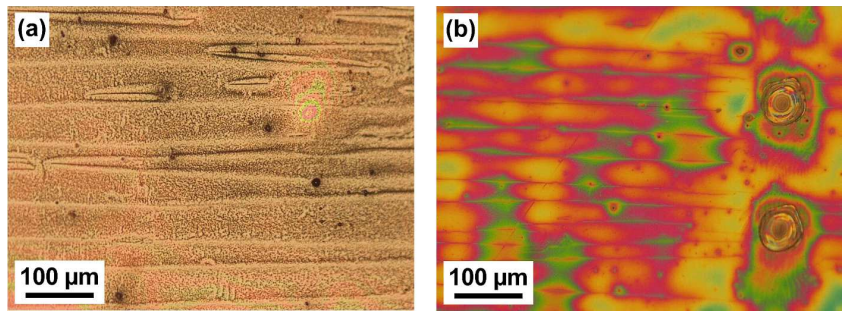

Fig. 6. The Bitter technique visualization of magnetic domains: (a) original sample without laser treatment,

(b) laser modified sample using a $5 \mathrm{~mJ}$ single pulse.

On the other hand, Fig. 7 shows the effect of $0.6 \mathrm{~mJ}$ single laser pulse regime on the magnetic domains visualized by MFM technique.
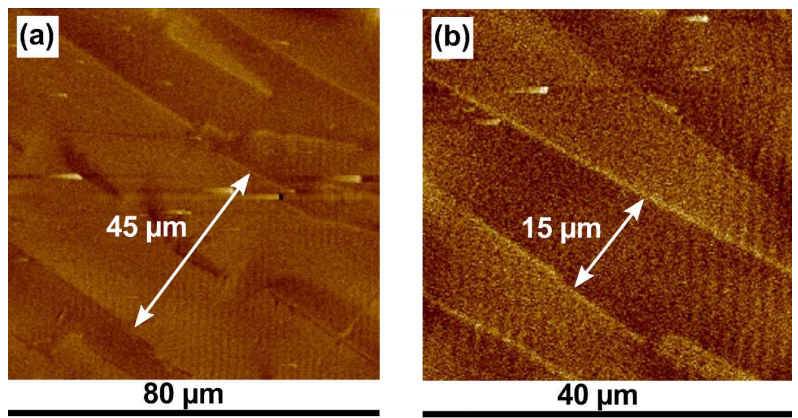

Fig. 7. MFM visualization of magnetic domains: (a) original sample without laser treatment, (b) laser modified sample using a $0.6 \mathrm{~mJ}$ single pulse.
Primary lamellar domains (Fig. 7a) were observed along the rolling direction which represents the easy magnetization direction. Obvious refinement in the domain width (Fig. 7b) was clearly observed in the grains between neighbouring laser spots. In accordance with findings of other authors [24-26], the domain refinement mechanism can be attributed to the effects of laser-induced residual thermal stresses and plastic deformation at the surface of the treated material. Figure 8 shows Kerr visualization of magnetic domains structures related to different laser pulse regimes using $0.6 \mathrm{~mJ}$ single pulse energy.
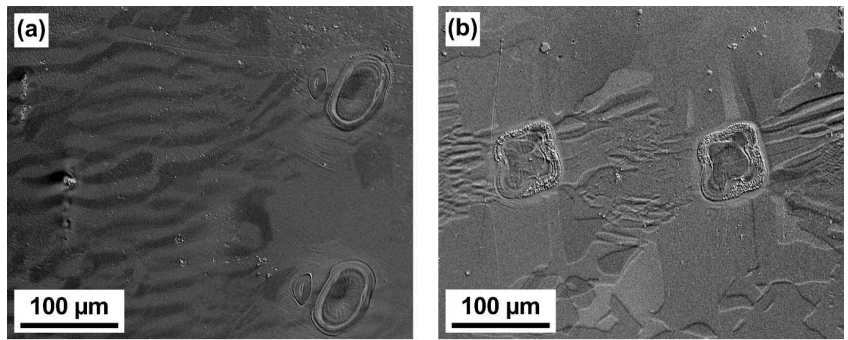

Fig. 8. Kerr microscopic visualization of magnetic domains structures obtained using $0.6 \mathrm{~mJ}$ single pulse energy within the regimes of: (a) single pulse, (b) 5 pulses.

The domain structures of single pulse (Fig. 8a) and multipulse regime with 5 pulses (Fig. 8b) exhibit favourable arrangements of refined primary domains and fine (non-detrimental) labyrinth subdomains in vicinity of laser spots. In addition, Fig. 9 shows SEM visualization of magnetic domains structures between neighbouring laser spots obtained by different laser pulse regimes using $0.6 \mathrm{~mJ}$ single pulse energy. The light lines indicate the positions of magnetic domain walls with perpendicular orientation to the laser scribe lines. The domain structures of single pulse (Fig. 9a) and 5-pulse regime (Fig. 9b) exhibit favourable arrangements of refined primary domains. The observed magnetic domain structures (Figs. 6-9) correlate well with obtained improvement in soft magnetic characteristics indicated by the decrease of coercivity values (Table II).
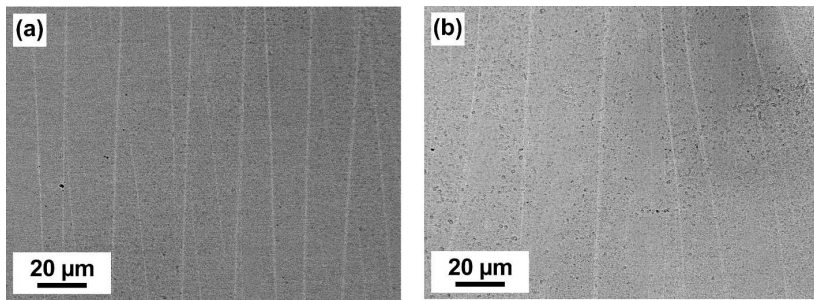

Fig. 9. SEM visualization of magnetic domains structures obtained using $0.6 \mathrm{~mJ}$ single pulse energy within the regimes of: (a) single pulse, (b) 5 pulses.

The present study has indicated that by optimization of laser pulse energy and pulse number at constant distance between individual laser spots, it is possible to improve soft magnetic characteristics of the studied material. 


\section{Conclusions}

Fiber laser pulse processing was carried out to investigate its effect on coercivity and nanohardness of $\mathrm{Fe}-3.2 \mathrm{Si}$ grain-oriented silicon steel in relation with observed surface changes and magnetic domains modifications. From the obtained results the following conclusions could be drawn:

- The single pulses with input energy values of $0.6 \mathrm{~mJ}$ and $5 \mathrm{~mJ}$ caused only a negligible melting effect on the material surface, whereas the use of $50 \mathrm{~mJ}$ single pulse caused a significant melting effect with high depth of penetration.

- With increase of number of pulses in multipulse laser regimes and introduction of pulse modulation, the material loss by ablation mechanism was increasing.

- The application of all fiber laser pulse regimes resulted in deterioration of local mechanical properties characterised by decreasing nanohardness values in centre of laser spots. With increasing pulse number and total supplied energy, the material damage at laser-treated surface was increasing.

- Clear improvements in soft magnetic characteristics i.e. remarkable decrease in coercivity values were obtained for the lowest single pulse energy of $0.6 \mathrm{~mJ}$ in all studied laser scribing regimes. Among them, a 5-pulse regime represents the most efficient laser treatment, optimized within the experimental conditions of present investigation.

- The observed improvement related to coercivity decrease depends not only on the primary (lamellar) domains refinement but also on the domain wall mobility influenced by the structure of secondary (labyrinth) domains. The optimal refinement and rearrangement of magnetic domains is determined by the balance of laser-induced residual stresses and plastic deformation at the surface of the treated material.

\section{Acknowledgments}

This work was carried out within the project, which is supported by the Slovak Research and Development Agency under the contract No. APVV-15-0259. This work was also partially supported by the Slovak Scientific Grant Agency VEGA, project No. 2/0081/16, No. $2 / 0120 / 15$ as well as by international project COST Action MP1401 and bilateral project SAV-AVCR 16-17. Also, the work was realized within the frame of the projects ITMS 26220220037, ITMS 26220220061, and ITMS 26220220064 .

\section{References}

[1] B. Weidenfeller, W. Riehemann, J. Magn. Magn. Mater. 292, 210 (2005).

[2] S.V. Ponnaluri, R. Cherukuri, P.A. Molian, J. Mater. Process. Technol. 112, 199 (2001).

[3] M. Ardebili, A.J. Moses, J. Magn. Magn. Mater. 112, 409 (1992).

[4] V. Mănescu, G. Păltânea, H. Gavrilă, G. Scutaru, Rev. Roumaine Sci. Techn. - Serie Électrotechn. Énergét. 60, 59 (2015).

[5] H. Wang, C. Li, T. Zhu, B. Cai, G. Huo, N. Mohamed, J. Mater. Sci. Technol. 29, 673 (2013).

[6] M. Takezawa, J. Yamasaki, T. Honda, C. Kaido, J. Magn. Magn. Mater. 254-255, 167 (2003).

[7] M. Enokizono, I. Tanabe, K. Takahashi, Y. Yoshida, T. Kubota, J. Magn. Magn. Mater. 196-197, 335 (1999).

[8] K. Sato, M. Ishida, E. Hina, in: Kawasaki Technical Steel Report, No. 39, 1998.

[9] P. Rauscher, J. Hauptmann, E. Beyer, Phys. Proced. 41, 312 (2013).

[10] S. Ahn, D.-W. Kim, H.S. Kim, K.-H. Baik, S.J. Ahn, C.G. Kim, Phys. Status Solidi B 241, 1641 (2004).

[11] L.A. Dobrzański, A. Drygała, J. Mater. Process. Technol. 191, 228 (2007).

[12] A.D. Compaan, I. Matulionis, S. Nakade, Opt. Lasers Eng. 34, 15 (2000).

[13] P. Švec, A. Schrek, M. Dománková, Kovove Mater. 54, 407 (2016).

[14] Q.G. Ma, X. Gao, J.Q. Li, Kovove Mater. 55, 45 (2017).

[15] V. Puchý, F. Kováč, P. Hvizdoš, I. Petryshynets, M. Sopko, High Temp. Mater. Proc. 35, 739 (2016).

[16] V. Puchý, F. Kováč, I. Petryshynets, L. Falat, Mater. Sci. Forum 891, 214 (2017).

[17] A. Hubert, R. Schäfer, Magnetic Domains, SpringerVerlag, Berlin 1998.

[18] M. Strečková, M. Batkková, I. Batkko, H. Hadraba, R. Bureš, Acta Phys. Pol. A 126, 92 (2014).

[19] D. Grüner, Z. Shen, J. Am. Ceram. Soc. 93, 48 (2010).

[20] P. Demir, E. Kacar, E. Akman, A. Demir, Acta Phys. Pol. A 125, 439 (2014).

[21] A. Zelenakova, P. Kollar, M. Kuzminski, M. Kollarova, Z. Vertesy, W. Riehemann, J. Magn. Magn. Mater. 254-255, 152 (2003).

[22] B. Weidenfeller, M. Anhalt, J. Magn. Magn. Mater. 322, 69 (2010).

[23] P. Rauscher, B. Betz, J. Hauptmann, A. Wetzig, E. Beyer, C. Grünzweig, Sci. Rep. 6, 38307 (2016).

[24] M. Imafuku, H. Suzuki, K. Akita, K. Iwata, M. Fujikura, Acta Mater. 53, 939 (2005).

[25] T. Nozawa, M. Mizogami, H. Mogi, Y. Matsuo, J. Magn. Magn. Mater. 133, 115 (1994).

[26] Y.N. Dragoshanskii, L.S. Karenina, V.D. Solovei, R.B. Puzhevich, Phys. Metals Metallography 112, 133 (2011). 